\title{
DBDigger: reorganized proteomic database identification improves flexibility and speed
}

\author{
David L. Tabb *† Chandrasegaran Narasimhan ${ }^{\text {* }}{ }^{\dagger \ddagger}$ Michael Brad Strader ${ }^{\S}$ \\ Robert L. Hettich ${ }^{\S}$ \\ January 14, 2005
}

Three items are included in this article's supplemental information:

- A sample search configuration file [search.ini] demonstrates the type of input required to configure a database search with DBDigger. While users currently edit these files by hand, a graphical user interface may be constructed to reduce errors for everyday use.

- Three spectra are shown [QSPVDI.pdf] for the peptide QSPVDI. Two spectra are for the intact peptide in two different charge states, and the third shows the spectrum for this peptide when ammonia is lost from the N-terminal Gln prior to precursor ion selection.

- Two spectra are shown [CKGTDVQAWIR.pdf] for the peptide CKGTDVQAWIR. The spectra differ by the addition of a modification with a mass of 42 Da near the $\mathrm{N}$-terminus.

\footnotetext{
*Corresponding author: (phone) 865 241-6006; (fax) 865 576-5332; (e-mail) tabbdl@ ornl.gov

${ }^{\dagger}$ Life Sciences Division, Oak Ridge National Laboratory, Oak Ridge, TN 37831-6164

${ }_{\ddagger}^{\ddagger}$ Genome Science and Technology, UT-ORNL Graduate School, Oak Ridge, TN 37830-8026

${ }^{\S}$ Chemical Sciences Division, Oak Ridge National Laboratory, Oak Ridge, TN 37831-6131
} 